\title{
Iatrogenic lumbar meningocoele: report of three cases
}

\author{
ITALO RINALDI AND THOMAS O. HODGES \\ From the Hampton Roads Neurological Center, Newport News, Virginia, and the Veterans Administration \\ Hospttal, Kecoughtan, Virginia, U.S.A.
}

SUMMARY We have reported three cases of iatrogenic lumbar meningocoeles after surg ery for herniated lumbar intervertebral discs. We reject the term spurious, pseudo, or false as given by earlier writers. We feel that, in reality, these sacs are true meningocoeles, with complete arachnoidal lining and freely communicating with the intraspinal subarachnoid space. We have also given a brief outline of the clinical features, elucidated the mechanisms operative in the production of symptoms, and suggested the cardinal features of radiographic diagnosis. Prevention is, of course, the best way to avoid this complication. If a dural tear does occur, every effort should be made to suture it in a watertight manner.

After surgery for herniated lumbar intervertebral discs, satisfactory remission of symptoms occurs in approximately 75 to $80 \%$ of cases. In evaluating those patients who have recurrence of symptoms, a few months or even many years after the original operation, one should not overlook a rare but easily diagnosable entity-the iatrogenic retrolaminar meningocoele. If at the time of surgery an accidental dura-arachnoidal tear occurs, either at the level of the root pouch or in the dural sac itself, spinal fluid will leak out of the subarachnoid space. If not properly handled, this will lead, in some instances at least, but certainly not too infrequently, to accumulation of fluid in the retrolaminar space artificially created by the dissection of the paravertebral musculature. Eventually a true meningocoele, with well-defined arachnoidal cell lining, will form. The fistulous opening, connecting the intradural subarachnoid with the extradural paraspinous space, may be small or large. In either event, at least some of these patients will experience recurrence of pain.

By now, it has become apparent that this complication is not too rare; indeed, several cases have already been reported in the literature. Nevertheless, in relation to the very large number of hemilaminectomies performed for the treatment of discal herniations, the iatrogenic meningocoele is still relatively infrequent and a rarely reported entity.

When we speak of post-operative meningocoeles, we do not mean at all to include the cases in which following a dura-arachnoid tear, a large amount of cerebrospinal fluid (CSF) will accumulate immediately after surgery. These are cases of massive leakage in which the walls of the fluid-containing cavity have no adequate time to become lined by arachnoidal cells. To these accumulations the term pseudo-meningocoele may be applied. In these cases more definitive surgery has to be carried out as soon as the complication becomes apparent and, indeed, one may actually see the skin bulging at the operative site. In the other cases, the ones with which this report is concerned, the sac goes unnoticed for months or years. These latter ones are true, albeit acquired, meningocoeles. In our opinion there is nothing spurious, false or pseudo about them, though these terms were used by Pagni, Cassinari, and Bernasconi (1961) and Miller and Elder (1968). They are true out-pouching of the dura-arachnoidal layers, freely communicating with the subarachnoid space, and undoubtedly capable of causing symptoms. Fortunately, if properly recognized, they can be treated very successfully in most instances.

\section{REVIEW OF LITERATURE}

Hyndman and Gerber, in 1946, reported two cases of acquired 'extradural cysts'. Both followed several months after bilateral laminectomies for tumours. The important point made in this paper was that the dural closures should be very tight and that leaving 
silver clips on the sutured margins of the dura should be avoided, since in one of their cases it did lead to a fistula and an extradural cyst.

Swanson and Fincher (1947) were the first to report three cases of 'extradural arachnoidal cysts of traumatic origin' after disc surgery carried out through the time-proven method of partial hemilaminectomy. They recognized, as a causal factor for these cysts, 'a dural laceration' and that failure to close this laceration properly had resulted in a persistent fistula. In each of their cases there was postoperative persistence or recurrence of symptoms not unlike those seen in individuals with a recurrent intervertebral disc herniation. Interestingly enough, in the first of their three cases, in view of the fact that this patient was involved in a compensation litigation, some doubt had been expressed as to the authenticity of his complaints, as in our third case.

The authors also mentioned the possibility of cyst formation, secondary to persistent cerebrospinal fluid leakage through single, or perhaps more often multiple, needle punctures of the dura-arachnoid for the purpose of myelographic studies.

They stressed that all dural lacerations must, if at all possible, be closed by the watertight method.

Winkler and Powers (1950) reported two cases and emphasized the fact that this condition may sometimes explain the 'persistence of symptoms in the low back following removal of a ruptured, intervertebral disc'.

One case was described in 1951 by Schreiber and Haddad. In this case the retrolaminar cyst communicated with the intraspinal space by way of a small pedicle which they ligated. Following excision of the cyst, there was complete remission of symptoms.

Viñas and Slade (1959) described one case in which a (bilateral?) decompressive laminectomy with excision of the L4 and L5 discs had been carried out. Months later, the patient noted recurrence of pain (radicular?). There was subsequent progressive intensification of pain to the point that the patient thought of suicide. Eventually the patient was readmitted and lumbar myelography disclosed a complete block at the L4 level. At re-exploration, a posterior meningocoele was found. The dural dehiscence was sutured.

Gurdjian, Webster, Ostrowski, Hardy, Lindner, and Thomas (1961) analysed 1,176 cases of lumbar disc operations performed by the usual method of partial hemilaminectomy. They recognized that if the dura is torn inadvertently, it should be repaired if necessary by an exposure through a more complete laminectomy. In their opinion and experience, gelfoam and/or muscle do not adequately control a dura-arachnoidal tear. Furthermore, they pointed out that 'extrusions of cauda equina filaments through a dural tear may cause radicular pain.' If such a tear is not repaired, a large internal meningocoele may form. They had to repair two such cases.

In 1961, one patient was reported in detail by Pagni et al. who applied to the fluid-containing meningocoele the term 'spurious'; to this term we no longer subscribe.

Shahinfar and Schechter, in 1966, referred to two cases of traumatic extradural cysts in which the dura had inadvertently been opened during lumbar disc surgery. They felt that this condition may be relatively asymptomatic or that perhaps, 'patients may accept some degree of discomfort' without subjecting themselves to re-examination. They also felt that another explanation for the rarity with which it is reported may be that post-operative myelography is not performed routinely as a long-term follow-up, implying that if this were done more cases would be discovered. These authors referred to other cases reported by Borgstrom, Kettler, Rosenblum, and Derow, and Strully and Heiser.

Lombardi and Passerini, in their 1964 monograph, referred to 'circumscribed ectasia of the dural sac' disclosed, on myelography, in three patients who had experienced recurrent symptoms and who had been previously operated upon for lumbar disc prolapse. Taveras and Wood (1964), as well as Shapiro (1968), do not make any specific reference to this entity, although they both mention the possibility that an acquired extradural cyst may either result from external trauma or following a laminectomy.

Miller and Elder (1968) reported nine patients in which the 'pseudocysts' were to be considered as a complication of lumbar hemilaminectomies and one as a complication of lumbar myelography. In one of their cases (case 10), the large cyst had developed over a two-month period after laminectomy, with fluctuant mass at the operative site. Interestingly enough, no dural tear had been recognized at the time of surgery. The time interval between the original hemilaminectomies and the time at which the cyst had been recognized ranged from two months to $9 \frac{1}{2}$ years.

Rinaldi and Peach (1968) described two instances of post-operative retrolaminar meningocoeles. Surgical excision resulted in complete remission of symptoms. The authors suggested that the diagnosis should be suspected in patients with recurrent radicular compression syndromes, especially if in the original operative note, mention had been made of a dura-arachnoid tear. They also suggested that 'the presence of extraspinal, retrolaminar Pantopaque on plain $x$-ray films may well be diagnostic of this complication'. They also re-emphasized watertight closure of any dural dehiscence at the time of the original surgery and described a method of repair. 
The first two cases were described in detail by Rinaldi and Peach (1969).

\section{CASE 1}

A 50-year-old man underwent surgery for a central disc herniation at the L3-L4 level in December 1960. While freeing the L4 root through a usual partial hemilaminectomy approach, a small tear occurred at the level of the axilla of the root. Cerebrospinal fluid leakage became apparent. Oxycel gauze was used to control the leakage, and healing was uneventful.

The patient did very well until May 1961, when he noticed recurrence of transient, radiating pain following the L4 root distribution. Pressure over the ipsilateral paraspinal musculature at the level of the previous hemilaminectomy reproduced the same pain which was indeed severe enough to make him fall. Plain radiographs revealed the presence of a drop of extra-spinal Pantopaque. This drop was most evidently lying within the meningocoele itself. However, at first, we had felt that this perhaps represented Pantopaque which had escaped along the needle tract after myelography.

Needle aspiration, at approximately $2 \mathrm{~cm}$ depth, yielded clear spinal fluid with a protein of $138 \mathrm{mg}$ per $100 \mathrm{ml}$. Twelve millilitres of Pantopaque were injected, and films clearly showed a voluminous meningocoele with a large fistulous canal connecting the sac with the intraspinal subarachnoid space (Fig. 1).

OPERATION The sac, which could easily be separated from the spinous processes medially and from the paraspinal musculature laterally and posteriorly, was found to be lying over the laminae of L3 and L4. Upon opening the sac, the fistulous canal was seen and a freely floating nerve root was identified. Complete resection of the meningocoele was then carried out except for a small section

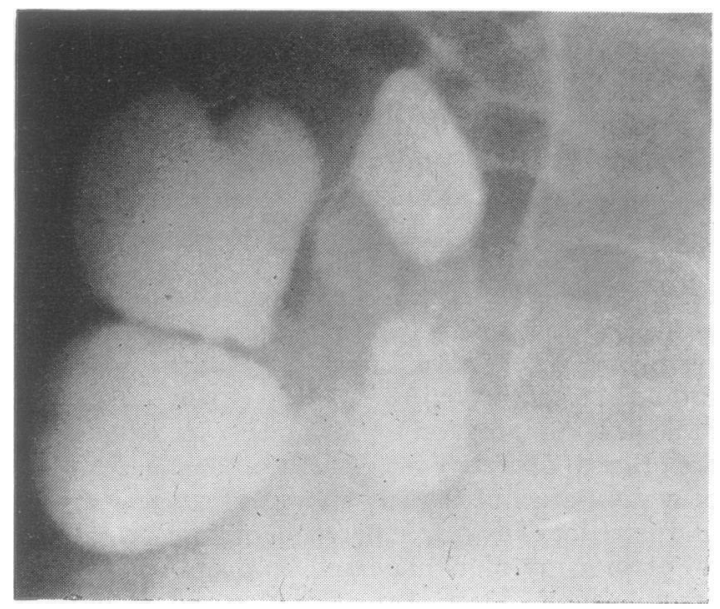

FIG. 1. Case 1. Note the large retrolaminar, paraspinous meringocoele extending over two laminae. which was shaped as a flap and reflected onto the fistulous opening and sutured to its margins, using 4-0 black silk sutures. Healing was uneventful and so far the patient has enjoyed complete remission of symptoms.

\section{CASE 2}

A 42-year-old woman had had two operations by another surgeon, the first in 1957, with excision of a herniated disc at the L5-S1 interspace on the left side. Because of recurrence of pain, in 1960, re-exploration was carried out. A dural tear occurred, and a small amount of Oxycel was used to control the cerebrospinal fluid leakage. After surgery the leakage persisted and, in fact, several days later the old incision had to be re-opened and 'a false meningocoele' was found. The operative report states that the dural tear could not be closed but tissues adjacent to the sac were used to close the defect by imbrication.

The patient continued to have occasional aches in the lower back and, in April 1968, she developed severe pain with radiations into both gluteal regions, most marked on the left side. A myelogram disclosed a large, paraspinal meningocoele on the left side. Again, the fistulous tract could be identified very readily (Fig. 2).

OPERATION The sac was found to be loosely adherent to the adjacent cicatricial tissue and could be dissected alt the way down to its neck, at the level of the interlamina
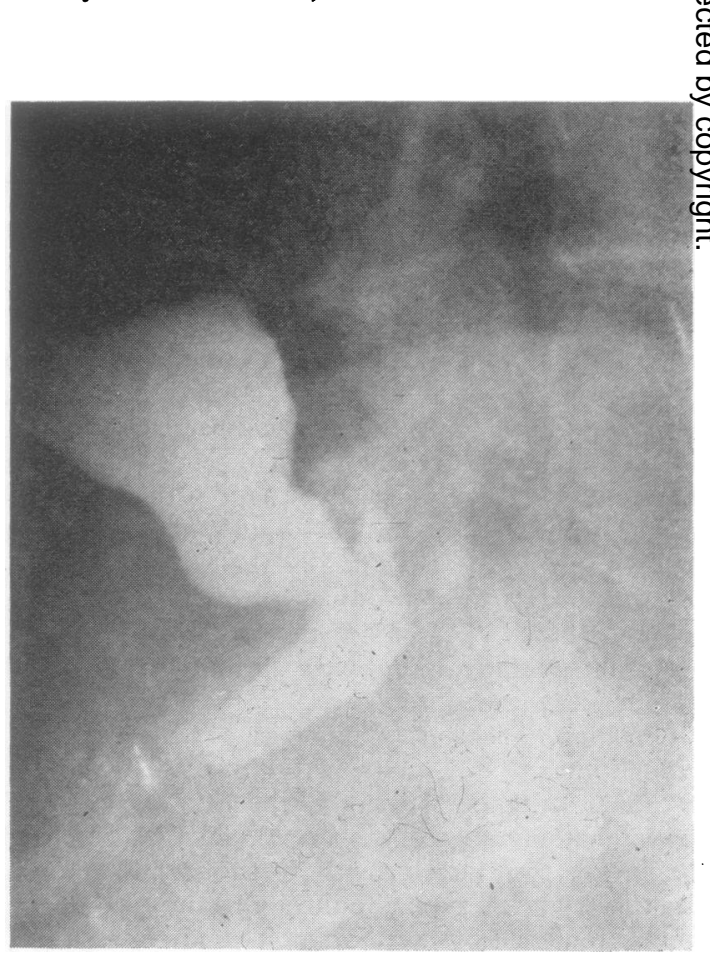

FIG. 2. Case 2. Cross-table lateral film in the supine decubitus. The sac is incompletely filled. The fistulous tract is clearly shown. 
space. The lining of the sac was white and glistening, and at the bottom a round opening was visible. Across the opening, a nerve root, which did not react to mechanical stimulation, was visible. The root was dissected free from the margins of the opening and allowed to drop back into the intraspinal subarachnoid space. The meningocoele was then excised except for a small portion which was sutured, as in the previous case, without difficulty.

The post-operative course was uneventful, with excellent remission of symptoms.

In June 1969, the patient sneezed and began to experience pain in the back and left leg. She was hospitalized in Macon, Georgia, and Dr. Charles H. Field performed a myelogram which clearly demonstrated complete obliteration of the meningocoele (Fig. 3).

\section{CASE 3}

A 49-year-old shipfitter was first seen in July 1965, because of recurrent pain in the lower back, radiating into the right buttock and posterior thigh, calf, and foot, aggravated by straining and bending. In 1959, he had been operated upon elsewhere for a herniated disc on the left side. The clinical findings were compatible with an S1 compression radiculopathy.

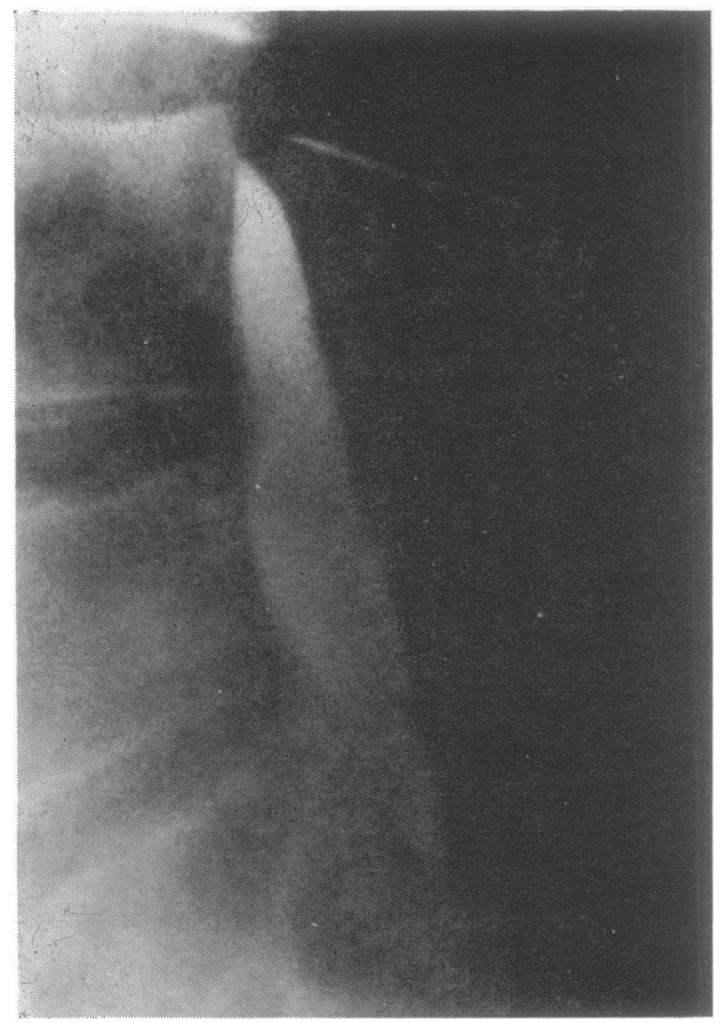

FIG. 3. Case 2. Myelogram 14 months after excision of meningocoele. Note smoothness of posterior dura at L5.
Radiographs revealed the presence of a fusion mass, extending from $\mathrm{L} 4$ to the upper sacrum, bilaterally. There was marked narrowing of the L5 interspace, as well as minimal narrowing of the L4 intervertebral space. A lumbar myelogram disclosed the presence of a complete extradural block at L4.

On July 15, exploratory surgery was carried out. A large pseudoarthrotic cleft was found at the L4-L5 level on the left side. In order to expose the dural sac, a roughly cylindrical portion of the fusion mass was removed from the midline at the L4-L5 level, with considerable difficulty. As this bone was being rongeured, it was apparent that a large section of the fusion mass was compressing the dura and its contents from a posterior direction. Furthermore, by careful extradural dissection, a large disc herniation at the L4 level was uncovered and removed. In trying to separate the dura from the underlying posterior longitudinal ligament, a small dura-arachnoidal tear occurred along the lateral portion of the dural sac on the right side. The leakage was controlled by using Oxycel and gelfoam.

Recovery was uneventful, and the patient did very well for eight months, after which he began to complain of discomfort in the lower back. Eventually, the pain radiated into the right lower extremity again. It was aggravated by standing, bending, and straining. Lying down relieved this pain very promptly.

The patient was admitted to the Veterans Administration Hospital in November 1968. Plain $x$-ray films revealed the irregularly cylindrical defect in the midline of the fusion mass. A few droplets of Pantopaque were seen outside the spinal canal proper, at this level (Fig. 4). A myelogram was carried out, and it revealed a sizeable meningocoele, fairly well centred in the midline and connected to the intraspinal subarachnoid space (Figs. 5 and 6 ). The entire extent of the sac could not be fully evaluated, but, nevertheless, the sac was quite large, and it extended about halfway posteriorly within the paraspinal muscular mass (Figs. 7 and 8). In the oblique view, a swollen nerve root could be seen to be in close approximation to the fistulous opening (Fig. 9). The spinal fluid protein content was $45 \mathrm{mg} / 100 \mathrm{ml}$.

Examination revealed a man who appeared to be in moderate discomfort and who seemed to be truthful about his statements, although it had been thought by many that his symptoms were mainly of a hysterical nature. Radicular pain could be elicited by the jugular compression manoeuvre as well as by pressure over the old operative scar. There was no evidence of bulging at this site.

At surgery, we were able to dissect the sac from the cicatricial tissue on its right side without difficulty. The dome of the sac was partially calcified, but again dissection could be accomplished without difficulty. On the left side, the wall of the sac could not be separated very readily, especially in its most anterior portion where it adhered to the bony fusion defect.

The dome was then cut into. The cavity was whitish and glistening. At its bottom, and slightly more to the right side, a roughly oval fistulous opening was clearly seen (Fig. 10). A nerve root, which appeared somewhat larger than usual and very much reddened, was actually herniating through the fistulous opening (Fig. 10, right). 


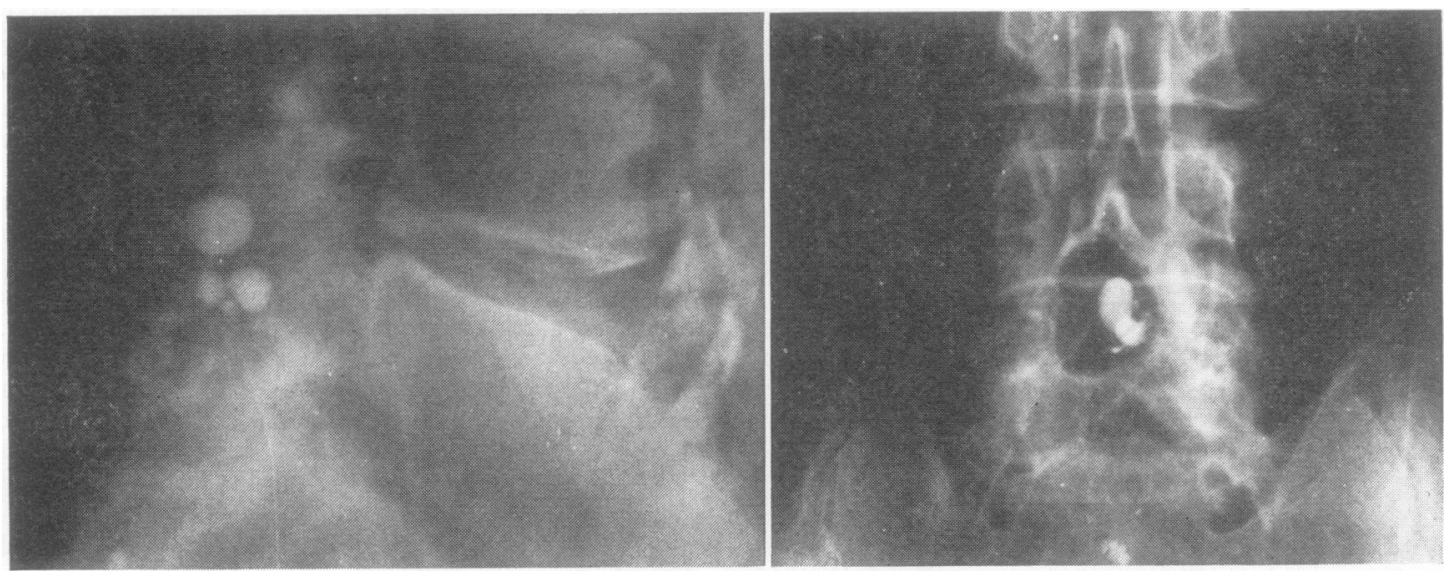

FIG. 4. Case 3. Pre-myelogram APand lateral supine films. Note the roughly round opening in the centre of the fusion mass. The Pantopaque droplets clearly lie outside the spinal canal. This we feel is pathognomonic of the post-operative meningocoele. The contrast medium could easily be 'returned' into the spinal subarachnoid space by placing the patient in a prone position.

Clear spinal fluid was freely draining into the cavity itself. The nerve root could be easily dissected from the lips of the fistulous opening to which it had been loosely adherent (Fig. 10, left). After it had been freed, it still showed a tendency towards herniating into the cystic cavity with each pulsation of the spinal fluid.

Closure of the fistula was rather difficult because the cystic wall at the level of the opening consisted only of a thin layer of arachnoidal cells, without any underlying

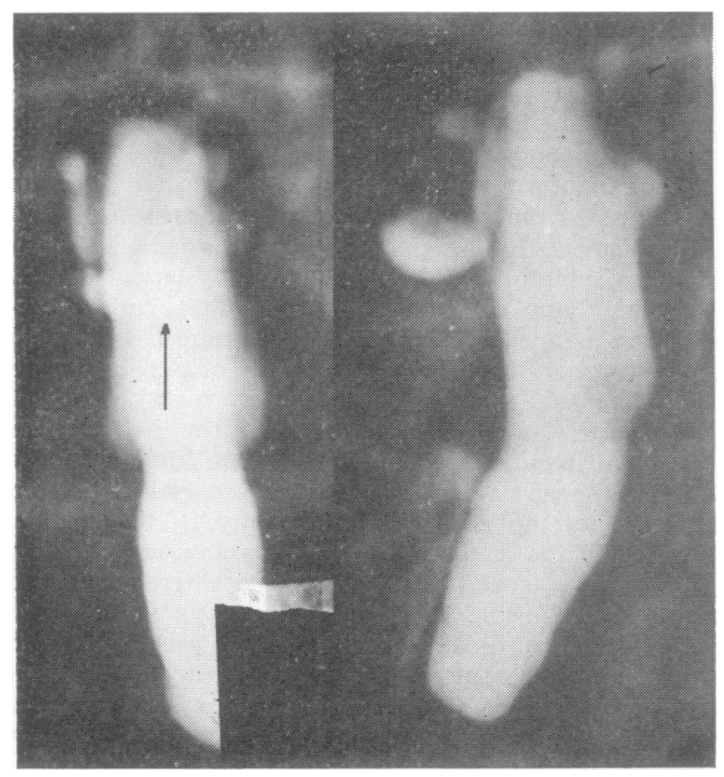

FIG. 5 membrane that one could utilize for the surgical repair. The membranous portion of the sac was excised. The posterior dura, which was creating the medial wall of the fistula, was then split without difficulty. This allowed us to use this small, fibrous dural flap to close the fistulous opening. The flap was hinged to the right and sutured, with some difficulty, to the lateral dura of the sac. The sutures did not completely obliterate the opening in a watertight manner, but definitely prevented herniation

FIG. 5. Case 3. AP film showing the lowermost extent of the sac (arrow) as a crescent of double density.

FIG. 6. Right oblique film showing the bottom of the sac and, above it, a small portion of the fistulous canal with a nerve root fixed to its intraspinal opening. 


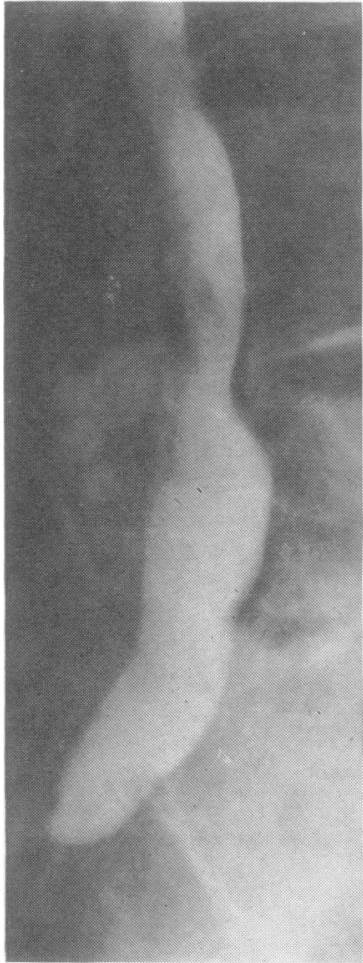

FIG. 7

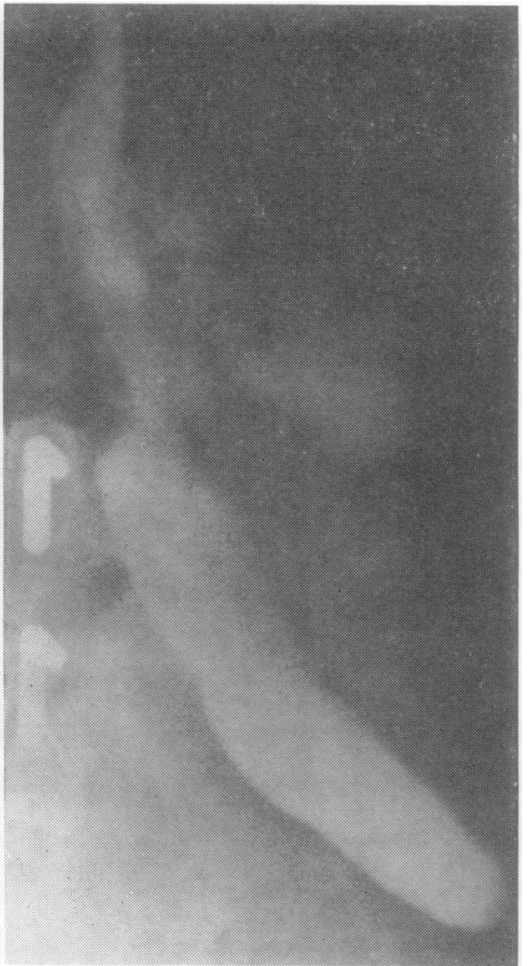

FIG. 8

of the nerve root. Two large stamps of muscle were then placed over the suture line. This seemed to establish a watertight closure, as well as obliteration of the bony portion of the cystic cavity.

The post-operative course was unremarkable. Subsequently, on June 23, the patient was re-examined, and at that time, he was ambulatory, intermittently complaining of some discomfort in the left buttock and posterior thigh, especially noted on bending, stooping, or straining. Repeat myelography, however, showed the presence of a chronic arachnoiditic process which most likely explains the persistence of some pain. Manipulation of the oil confirmed the complete obliteration of the extraspinal cyst (Fig. 11). Oral steroids have helped the discomfort remarkably.

\section{CLINICAL FEATURES AND MECHANISMS OF PAIN}

Meningocoeles after surgery for discal herniations have, in our experiences, as in those of all other authors, been symptomatic. Undoubtedly, in certain instances, it is conceivable that a meningocoele may be present without producing sufficient discomfort to induce the patient to be re-examined. In most - cases, however, there will be recurrence of low back pain, at times only periodically. Frequently, if not always, there will be recurrence of the radicular

FIG. 7. Case 3. Cross-table lateral film in the supine position, demonstrating the fistulous canal and a small portion of the meningocoele.

FIG. 8. A larger amount of Pantopaque has entered the extraspinal sac.

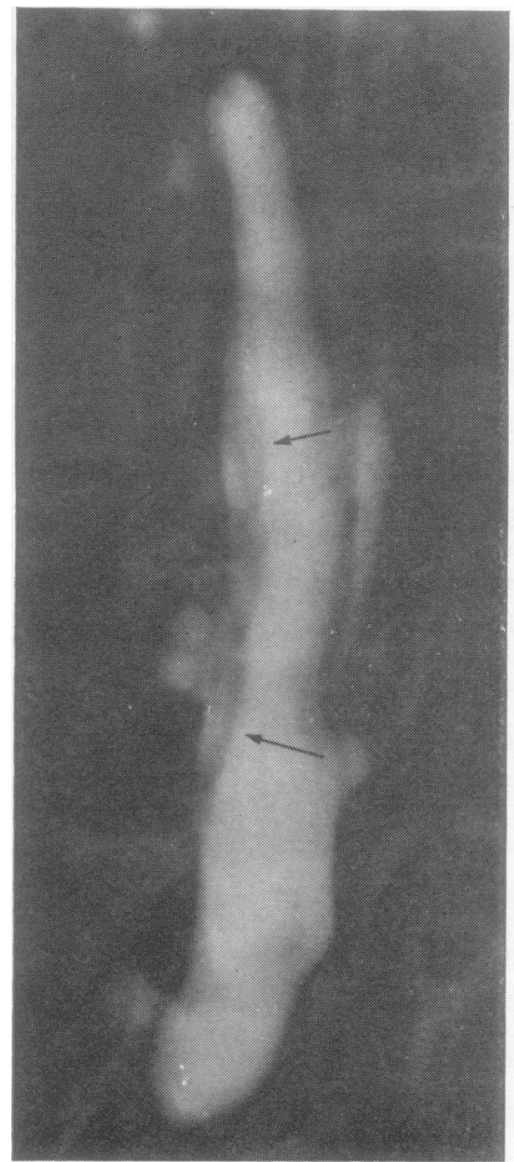

FIG. 9. This film shows the relationship of the nerve root to the intraspinal portion of the fistula. 

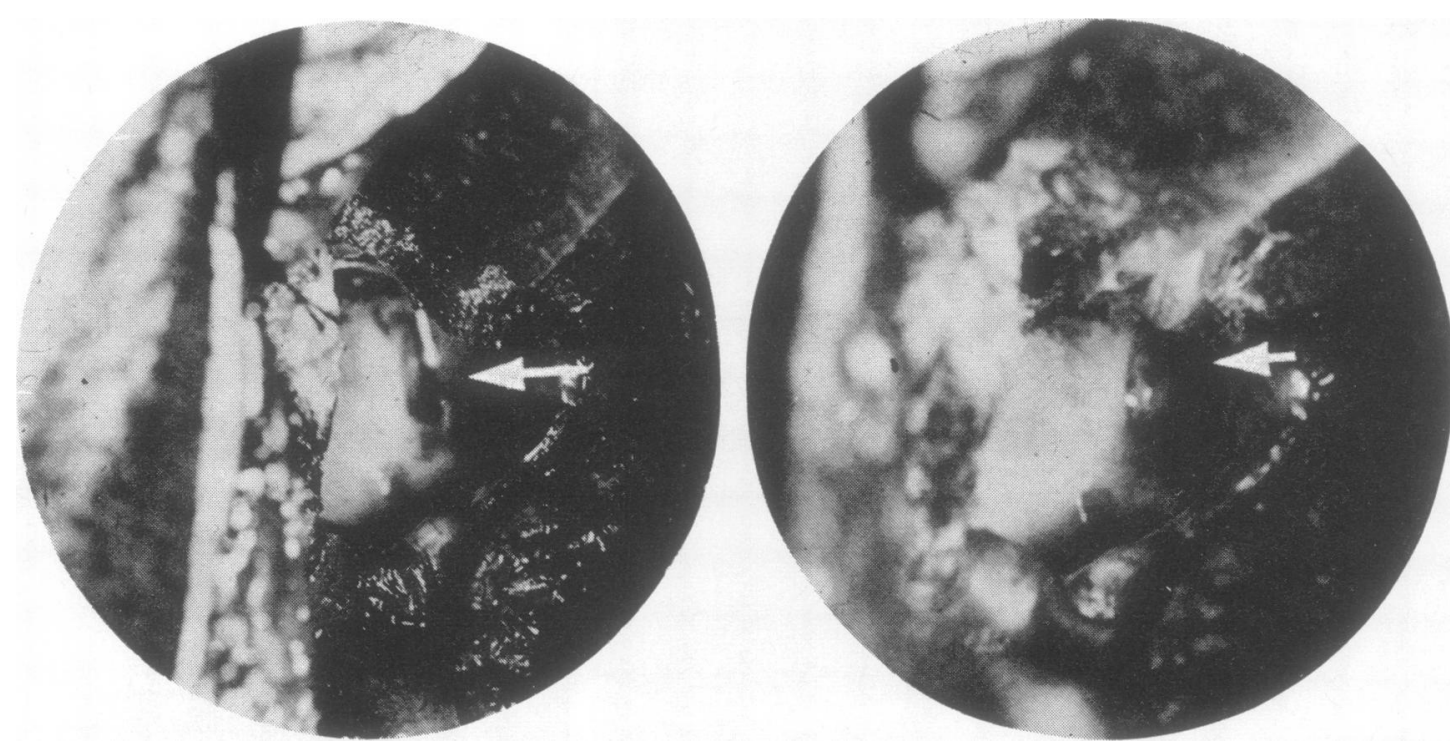

FIG. 10. This is the first surgical photographic demonstration of an iatrogenic extraspinal meningocoele. We think that it $\omega_{\text {W }}$ conclusively illustrates the adherence of a nerve root (arrow) to the opening connecting the intraspinal subarachnoid space it with the extraspinal meningocoele. Note the oedematous root bulging into the sac. The photograph on the left was take ${ }_{6}$ after the root had been teased off the opening and allowed to drop back in its natural location. Note the now empt fistulous opening!(arrow).

irritation syndrome, at whichever level the original surgery had been carried out. Very often the radicular pain has been precipitated, or aggravated, by straining or by any other manoeuvres which increase intracranial and intraspinal pressure, such as coughing, sneezing, or jugular compression. In some cases, as in our first one, manual pressure over the paraspinal musculature on the side of the cyst would reproduce the pain. In our third case, as in one patient described by Swanson and Fincher (1947), the symptoms were at first regarded as being on a psychoneurotic basis until myelography, and eventually surgery, disclosed the real nature of the problem.

We feel that at least two mechanisms are operative in the production of symptoms.

1. As clearly shown in our cases 2 and 3 , a nerve root may become quite adherent to the fistulous opening. It is easy to understand how either movements of the spine, pressure over the paraspinal musculature, or straining would cause traction or stretching of the anchored nerve root. We cannot but disagree with the statement made by Miller and Elder (1968) that, as in their cases 1 and 2, in which a ... filament of the cauda equina was adherent to the opening in the dura ...' yet, these 'adhesions did not appear to be the cause of symptoms'! What then?
2. A nerve root (or perhaps even more than one, if the fistulous opening is large enough) may actuall herniate, at least temporarily, into the extraspinats sac. We have seen this demonstrated, to our com= plete satisfaction, in cases 1 and 3. This mechanism was also operative in the cases described by Kettler and Strully. When this occurs, it is straining that will cause pain by wedging the nerve root, or roots, into the fistulous opening with force.

There is no apparent relationship between the severity of pain and the size of the sac or of the fistula. The time interval between the original surgery and the recurrence of symptoms may range from a few months to several years.

\section{DIAGNOSIS}

First of all, it is necessary to have recorded in the operative report that a dura-arachnoidal tear has occurred. This would certainly arouse the suspicion of the surgeon that the recurrent symptoms may be related to the formation of a post-operative extradural meningocoele. Keeping this in mind, the presence of extraspinal retrolaminar Pantopaque in the plain radiographs of the spine may well be diagnostic of this complication. This was demonstrated retrospectively in our first case and in a much 


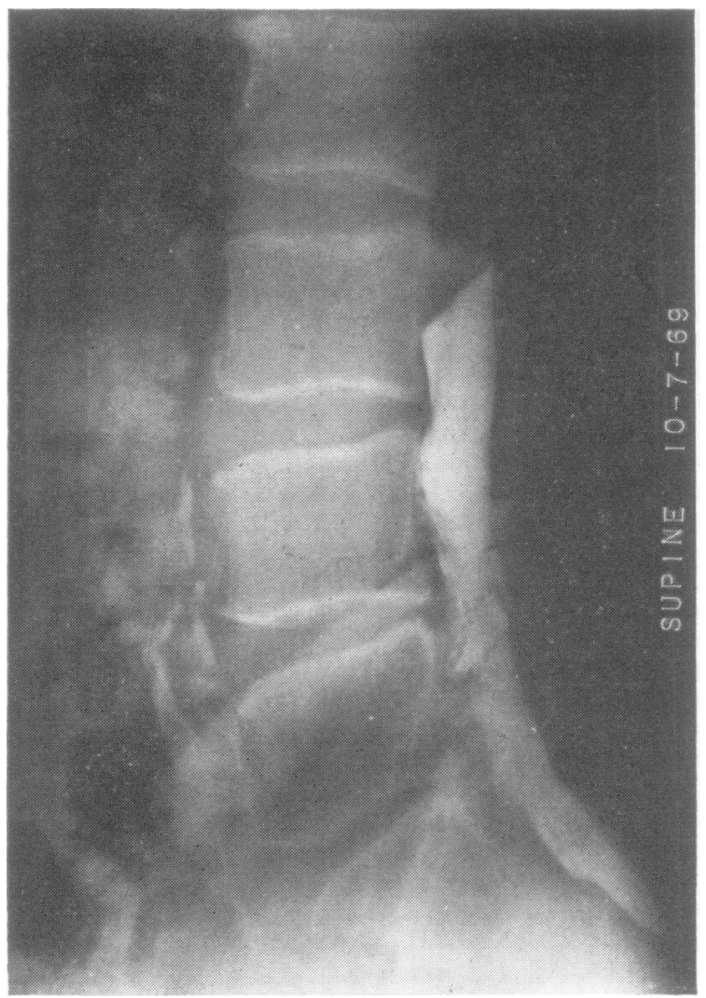

FIG. 11. Case 3. Myelography seven months after surgery revealed chronic arachnoiditis. This cross-table lateral film, in the supine position, with head tilted $45^{\circ}$ also confirmed the obliteration of the iatrogenic extraspinal cyst.

more clear fashion in our third case, where Pantopaque was seen to lie in the extraspinal space, at the operative site. Furthermore, we feel that if there is some contrast medium within the intraspinal subarachnoid space, it is wise to fluoroscope the patient and try to manoeuvre the oil outside the spinal canal. This can be more readily done with the patient in the supine position and perhaps in a slight lateral decubitus, on the side of the hemilaminectomy.

If a mass can be palpated in the operative area, Pantopaque may be directly injected into it percutaneously. Swanson and Fincher (1947), Shahinfar and Schechter (1966), as well as Rinaldi and Peach (1969), describe cases in which, while attempting to puncture the lumbar subarachnoid space for myelography, they penetrated the cystic cavity itself, into which the contrast material was then introduced. The spinal fluid proteins may be normal or markedly increased, as in case 1 of Rinaldi and Peach, in which the proteins were $138 \mathrm{mg} / 100 \mathrm{ml}$.
Myelography, in the usual manner, is otherwise essential in establishing the correct diagnosis, remembering, nevertheless, that it is necessary to carry it out not only in the prone position, but also in the supine and lateral decubitus, since the cysts are located on the dorsal aspect of the spinal canal.

With myelography, it is possible to determine (1) the presence of the extraspinal cystic cavity, (2) its actual rostral-caudal extent (it may cover one or two, or even more laminae), (3) the fistulous tract, and (4) the nerve root or roots adherent to the fistulous opening.

\section{TREATMENT}

First of all, it is important to prevent the formation of these meningocoeles by tightly suturing all the dural lacerations that may occur at the time of hemilaminectomies performed for the treatments of lumbar disc herniations, whenever possible. As first noted by Swanson and Fincher (1947), and later reiterated by Gurdjian et al (1961), as well as by Rinaldi and Peach (1969), gelfoam and/or muscle are poor substitutes for a careful, watertight suture.

Once the diagnosis of this complication is made, every effort should be made to close the fistulous opening in a watertight manner, even if it requires a more expanded laminectomy to do so. We feel that, whenever possible, the sac should be excised, except for a small portion, which can be shaped in the form of a flap, as suggested by Rinaldi and Peach (1969), and sutured with 4-0 or 5-0 silk to the margins of the fistulous opening. If a nerve root is found to be adherent to the opening, it should be gently teased away and allowed to drop into the intraspinal subarachnoid space.

\section{REFERENCES}

Gurdjian, E. S., Webster, J. E., Ostrowski, A. Z., Hardy, W. G., Lindner, D. W., and Thomas, L. M. (1961). Herniated lumbar intervertebral discs-an analysis of 1176 operated cases. J. Trauma, 1, 158-176.

Hyndman, O. R., and Gerber, W. F. (1946). Spinal extradural cysts, congenital and acquired. Report of cases. J. Neurosurg., 3, 474-486.

Lombardi, G., and Passerini, A. (1964). Spinal Cord DiseasesA Radiologic and Myelographic Analysis. Pp. 161-170. Williams and Wilkins: Baltimore.

Miller, P. R., and Elder, F. W. (1968). Meningeal pseudocysts (Meningocele spurius) following laminectomy. Report of 10 cases. J. Bone J. Surg., 50A, 268-276.

Pagni, C. A., Cassinari, V., and Bernasconi, V. (1961). Meningocele spurius following hemilaminectomy in a case of lumbar discal hernia. J. Neurosurg., 18, 709-710.

Rinaldi, I., and Peach, W. F., Jr. (1969). Post-operative lumbar meningocele. Report of two cases. J. Neurosurg., 30, 504-507.

Schreiber, F., and Hađdad, B. (1951). Lumbar and sacral cysts causing pain. J. Neurosurg., 8, 504-509. 
Shahinfar, A. H., and Schechter, M. M. (1966). Traumatic extradural cysts of the spine. Amer. J. Roentgenol., 98, 713-719.

Shapiro, R. (1968). Myelography. 2nd ed. Year Book Medical Publishers: Chicago.

Swanson, H. S., and Fincher, E. F. (1947). Extradural arachnoidal cysts of traumatic origin. J. Neurosurg., 4, 530-538.
Taveras, J. M., and Wood, E. H. (1964). Diagnostic Neuroradiology. Williams and Wilkins: Baltimore.

Viňas, F. J., and Slade, H. (1959). Meningocele como complicacion de laminectomía. Rev. méd. Cordoba, 47, ڤ 470-473.

Winkler, H., and Powers, J. A. (1950). Meningocele following hemilaminectomy: Report of two cases. N.C. med. J., 11, 292-294. 